\title{
Localization in Wireless Sensor Network based on Multidimensional Scaling (MDS)
}

\author{
Shraddha Singh \\ Dept. of Information and Communication Technology \\ Gautam Buddha University \\ Greater Noida, India \\ Dr. Vidushi Sharma \\ Dept. of Information and Communication Technology \\ Gautam Buddha University \\ Greater Noida, India
}

\begin{abstract}
Wireless Sensor Network (WSN) is a wide area used for different applications in today's scenario. Knowing the position of each node in the sensor network is very essential. Wireless sensor network is useless without localization information about each sensor node. Using GPS to find the position of sensor node is costly and power consuming solution. Also, it is not useful for indoor applications. So, we need to use localization algorithms. In this paper, we located the positions of unknown nodes in wireless sensor network by implementing an algorithm based on multidimensional scaling (MDS) technique. Distance matrix is calculated using different methods. To increase accuracy we have used distance approximation to refine distance matrix. We examined the performance of our algorithm for different network topologies and parameters by conducting simulations. From simulation we conclude that the algorithm performs well in terms of accuracy.
\end{abstract}

Keywords - wireless sensor network (WSN), localization techniques, multidimensional scaling (MDS)

\section{INTRODUCTION}

A Wireless Sensor Network (WSN) is the combination of small, low cost sensor nodes connected by wireless media [1]. These sensor nodes interact with each other to perform specific application. Nodes can sense and monitor the environment, compute the data and provide it to the users through base station. Due to its low cost and low power consumption, the WSN is used widely. WSN is used in military for battlefield surveillance, traffic monitoring, habitat monitoring, environmental monitoring etc. [2].

After monitoring the environment, sensor nodes process the data and circulate it through the network to the base station. Since, nodes are deployed in wide area and have short communication range, data cannot be sent directly to the base station. Every node transfers data to its closest node which further retransmits the packets. A node must know its location before transmitting data to its neighbors. An individual node must send location information along with the data collected in order to provide correct position to the users. This means that sensor node must localize itself [1].

The node localization problem is present in large number of WSN applications. The information collected from the network may be useless if it does not contain location information. Measuring the exact physical position of nodes is needed for continuous operation of wireless sensor network. Global Positioning System (GPS) can be equipped on the sensor nodes to estimate their position, but this solution is costly in terms of power consumption and money. Also, it is not useful for indoor applications.

Different localization algorithms have been developed for sensor networks. Most of them make use of some anchor nodes which know their position either by using GPS or being configured manually. The other nodes which do not know their location are called unknown nodes. The anchor nodes help the unknown ones to localize themselves [2]. The performance of localization algorithm depends on different parameters like the radio range, anchor nodes, the network topology, the density of nodes, etc.

Multidimensional scaling (MDS) is a form of data analysis techniques that has been used for a long time in mathematical psychology, marketing, economic research etc. MDS can make the data more understandable by representing it graphically. The data is grouped based on certain parameters. The dimensionality of data can be reduced using multidimensional scaling. MDS also works well in sensor network domain. It uses the distance 
information between nodes to calculate the coordinates of nodes in a $2 \mathrm{D}$ or $3 \mathrm{D}$ space. This distance information between the nodes is used as a data input. A distance matrix containing the distances between each pair of nodes in the network is created. MDS is a centralized method which can be used for the estimation of both relative and absolute position. All the data is gathered at sink node which perform further computation. MDS has the ability to recreate the relative map of the sensor network even when no anchor node is used. MDS estimate the position very accurately if sufficient number of anchor nodes is used by transforming local map into an absolute map.

In this paper, we analyzed classical multidimensional scaling method for localization in WSN. We investigated MDS-MAP in which distance matrix is calculated using Dijkstra's algorithm. We have also calculated the distance matrix using minimum path spanning tree algorithm. To reduce the errors in distance matrix we used a variation in MDS-MAP. To increase accuracy of the algorithm, heuristic method for calculating distance matrix is used. This improved algorithm is known as Improved Multidimensional Scaling (IMDS). We compared IMDS with Dijkstra's and spanning tree based MDS-MAP.

The remaining paper is organized as: the second section discusses the earlier work related to localization techniques. Section three discusses MDS based approach for localization. Section four discusses the results that are obtained from simulation. Lastly, we conclude in the fifth section.

\section{RELATED WORK}

Location information is of great importance for various wireless sensor network applications. Quick and accurate self localization capability is largely required in wireless sensor network. A number of localization algorithms have been developed with different logic. We can divide them into two types: range based and range free algorithms. The range based methods calculate the distance between neighboring nodes, and then use this data to localize nodes. Range free methods use estimated distance between nodes to localize nodes.

\section{A. Range-based Localization}

This technique estimates the actual distance between the neighboring nodes and then uses trilateration or triangulation to estimate the location of unknown node. Received Signal Strength Indicator (RSSI) [3] is the most basic technique that measures the received signal strength. Time of Arrival (TOA), Angle of Arrival (AOA), Time Difference of Arrival (TDOA) are some popular range based techniques [2]. These techniques provide higher accuracy but require additional hardware and thus the size and cost increases.

\section{B. Range-free Localization}

This technique estimates the position of unknown nodes on the basis of information provided by the anchor nodes. The information provided is usually the number of hop count or radio coverage [1]. This technique is also known as "hop-based" technique. Hop Count, DV-Hop, APIT and Centroid are some of the range-free techniques. These are less accurate than range based methods.

Multidimensional Scaling (MDS) based techniques are range based localization techniques. Different kinds of MDS techniques are available for localization. Classical MDS is the simplest MDS technique. It uses one matrix of similarity or dissimilarity as distance information. It uses this matrix to estimate the coordinates of unknown nodes. MDS-MAP is the most popular technique [4][8]. It is based on classical multidimensional scaling. It outperforms other localization techniques. MDS-MAP performance improves as the range improves. MDS-MAP contains the following steps-

i. The shortest distance between each pair of nodes in the network is calculated (by making use of Dijkstra's or Floyd's shortest path algorithm) and stored in a distance matrix.

ii. The classical MDS is applied on distance matrix. Relative map for each node is created by calculating initial two eigen vectors and eigen values.

iii. By using anchors, relative map is transformed into absolute map.

Other MDS based techniques are also available but they are more complex. MDS MAP (P) and MDS-MAP (P, R) are based on MDS-MAP that work well in both uniform and irregular networks [4][5]. These algorithms use the distributive approach. Each node simultaneously calculates its relative map using its two- hop neighbors. These maps are then merged together to form the global map using the anchors. MDS-MAP (P, R) refines the global map of MDS-MAP(P) using the least square minimization [4][5]. This is an improvement to MDS-Map (P). 


\section{MDS BASED APPROACH FOR WSN LOCALIZATION}

In MDS based localization algorithms distances between non-neighboring nodes is estimated to create a distance matrix. MDS can be very accurate if correct distance matrix is given as input. The distance matrix can be created using different methods. In MDS-MAP distance matrix is created using Dijkstra's or Floyed's algorithms for shortest path. It can also be created using minimum spanning tree algorithm. Distance matrix created using these algorithms is not correct. The distance estimated by these algorithms correlates with eucledian distance, but it is always the longest possible distance.

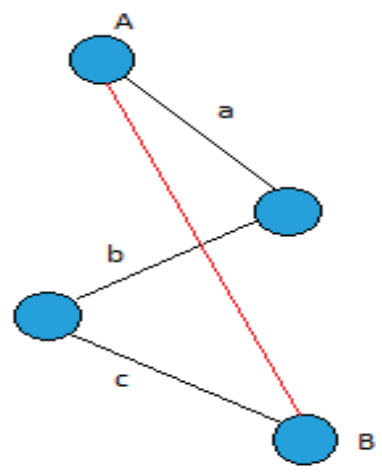

Fig. 1: Distance Calculation through Dijkstra Algorithm

\section{A. Dijkstra's Algorithm for distance matrix estimation}

In WSN, only the distance between neighboring nodes is known. So, to calculate the distance between the nodes which are not in direct contact Dijkstra's algorithm is used in MDS-MAP. Dijkstra's algorithm which is graph search algorithm can solve the shortest path problem for single source. We represent the sensor network as graph which has non-negative edges path cost and the eucledian or real distance between non neighboring nodes is the Dijkstra distance. But, the assumption that eucledian (real) distance correlates with the Dijkstra distance is hardly true. This assumption introduces an error such that the distance calculated by Dijkstra's algorithm between non neighboring nodes is much larger than the actual distance. Fig. 1 shows that the distance estimated by dijkstra' algorithm between nodes $\mathrm{A}$ and $\mathrm{B}$ is $\mathrm{AB}=\mathrm{a}+\mathrm{b}+\mathrm{c}$, which longer than the actual distance The estimated error is even greater when the nodes are present in multi-hop communication range.

\section{B. Minimum Spanning Tree Algorithm for distance matrix estimation}

Minimum spanning tree algorithm can also be used for distance matrix estimation. Minimum spanning tree is a tree in edge-weighted graph such that its weight is minimum in all the spanning trees of that graph. A spanning tree connects all the vertices of that graph. As similar to Dijkstra's algorithm, the spanning tree algorithm also estimates longer distance between the non-neighboring nodes than the actual distance. This introduces error in distance matrix. Thus, the location estimation using MDS is not accurate.

\section{Heuristic Approach for distance matrix calculation}

To reduce the error introduced in distance matrix, we use a heuristic approach to calculate distances between nonneighboring nodes as proposed in [7]. Let us consider that a WSN contains three nodes, (A, B and C) as shown in Fig. 2. The distances between A and B (d1), and B and C (d2) are known. Since distance matrix needs the distance between each node pair, the distance between $\mathrm{A}$ and $\mathrm{C}$ which are not in direct contact need to be calculated. 


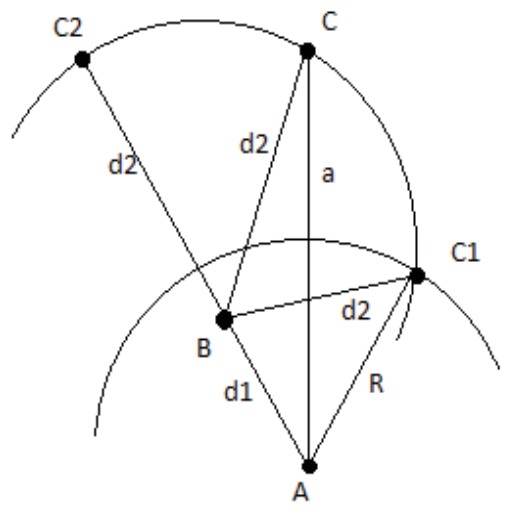

Fig. 2: Heuristic Approach based distance approximation

If the maximum radio range of nodes is $\mathrm{R}$, then node $\mathrm{C}$ lies on the curve $\mathrm{C} 1 \mathrm{C} 2$. Let us assume that $\mathrm{C}$ lies in the mid of curve $(\mathrm{C} 1 \mathrm{C} 2)$ then the distance between $\mathrm{A}$ and $\mathrm{C}$ can be estimated using cosine formula-

$$
\begin{aligned}
a^{2} & =d_{1}{ }^{2}+d_{2}{ }^{2}-2 d_{1} d_{2} \cos (\Varangle A B C) \\
& =d_{1}{ }^{2}+d_{2}^{2}-2 d_{1} d_{2} \cos \left(1 / 2 \Varangle A B C_{1}\right)
\end{aligned}
$$

Where, $A B C_{1}=\arccos \left[\left(d_{1}^{2}+d_{2}^{2}-R^{2}\right) / 2 d_{1} d_{2}\right]$

\section{SIMULATION RESULTS}

We assumed that a wireless sensor network containing 100 sensor nodes distributed over two-dimensional 100x100 monitored area. Sensors contain omni-directional antennas. Sensor nodes have maximum radio range R and can communicate with the nodes that are present within this radio range. We have assumed that a path is always available between each node pair and nodes in proximity to each other can exchange messages. Also, RSSI technique is used for distance estimation between neighboring nodes.

We mainly considered grid and random topology for our work. We have assumed no radio range error present in the network. We implemented MDS-MAP ( Dijkstra's), MDS-MAP ( Spanning tree) and IMDS techniques using Matlab. We compared the results for these techniques. We considered different network parameters like connectivity, number of anchor nodes and estimated error. We have simulated the results using 4,8,12 and 15 anchors for both grid and random topology. Thus, we simulated different networks and discovered each node location using MDS-MAP (Dijkstra’s), MDS-MAP (Spanning tree) and IMDS techniques.

The error estimated through these techniques is normalized by using the radio communication range (R) as proposed in [5][7]:

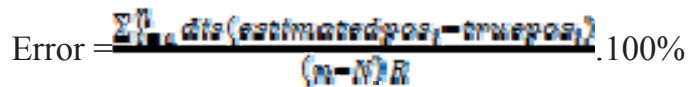

Where,

$\mathrm{N}=$ number of anchor nodes,

$\mathrm{n}=$ number of sensor nodes in WSN,

estimatedPos $\mathrm{i}_{\mathrm{i}}=$ estimated position of $\mathrm{i}^{\text {th }}$ node,

truePos ${ }_{\mathrm{i}}=$ true position of $\mathrm{i}^{\text {th }}$ node 


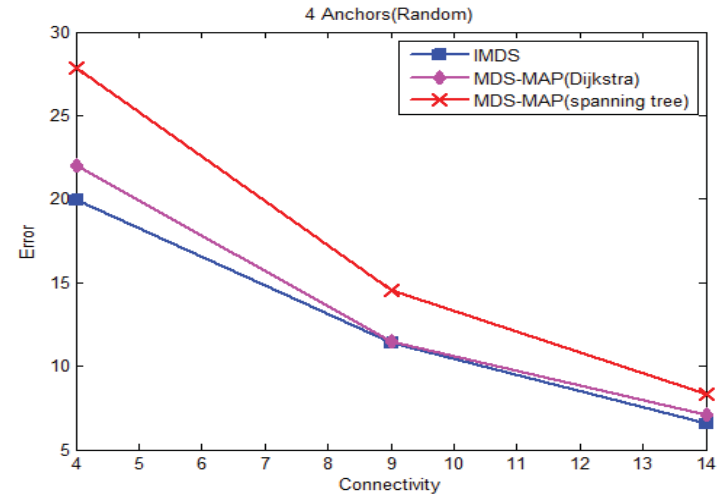

Fig. 3: Error versus Connectivity graph for Random Topology ( using 4 anchors)

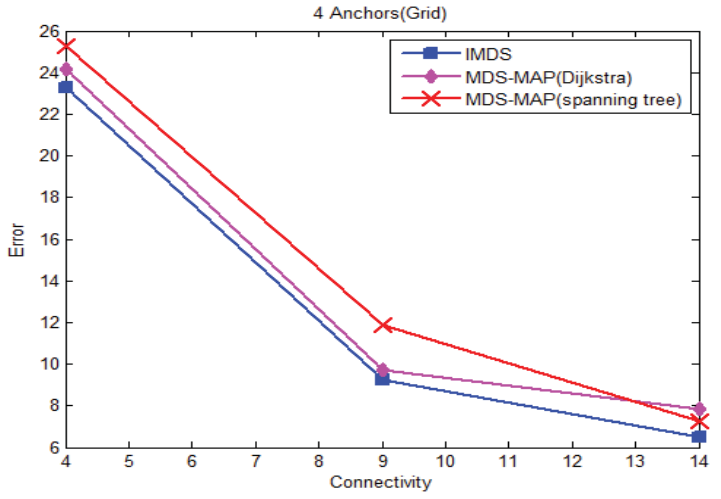

Fig. 4: Error versus Connectivity graph for Grid Topology ( using 4 anchors)

Fig. 3 and Fig. 4 show the comparison of IMDS, MDS-MAP (Dijkstra) and MDS-MAP (Spanning tree) using 4 anchors for random and grid topology respectively.

Fig. 5 and Fig. 6 show the comparison of IMDS, MDS-MAP (Dijkstra) and MDS-MAP (Spanning tree) using 12 anchors for random and grid topology respectively.

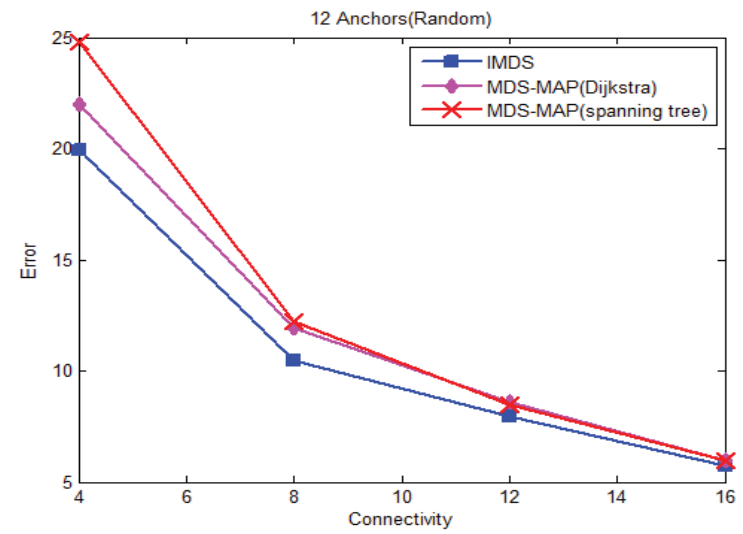

Fig. 5: Error versus Connectivity graph for Random Topology ( using 12 anchors)

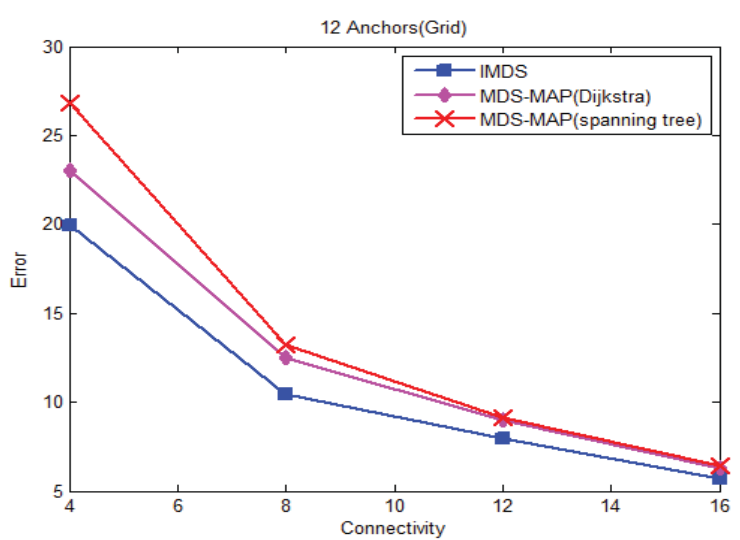

Fig. 6: Error versus Connectivity graph for Grid Topology( using 12 anchors

From the figures, we say that IMDS shows better results than MDS-MAP. It introduces less estimation error for both grid and random topologies.

Fig. 7 shows the network map estimated using IMDS technique for random topology. We can see from the figure that the deviation in estimated and actual location is very small.

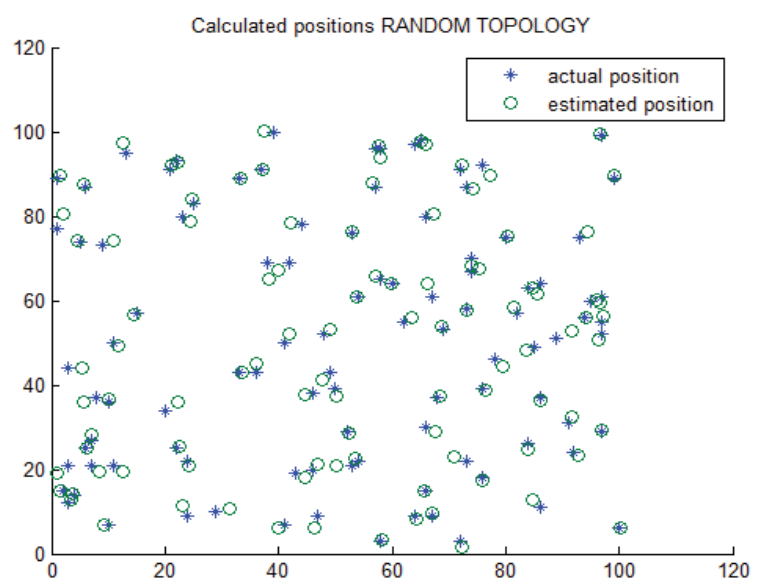

Fig. 7: Network map estimated using IMDS (Random Topology) 
Fig. 8 and Fig. 9 show the impact of number of anchor nodes on the estimated error for random and grid topology respectively. These networks do not have range error. Figure show that the localization error is reduced by using more anchor nodes. The impact of anchor nodes is not significant when density of the network is high.

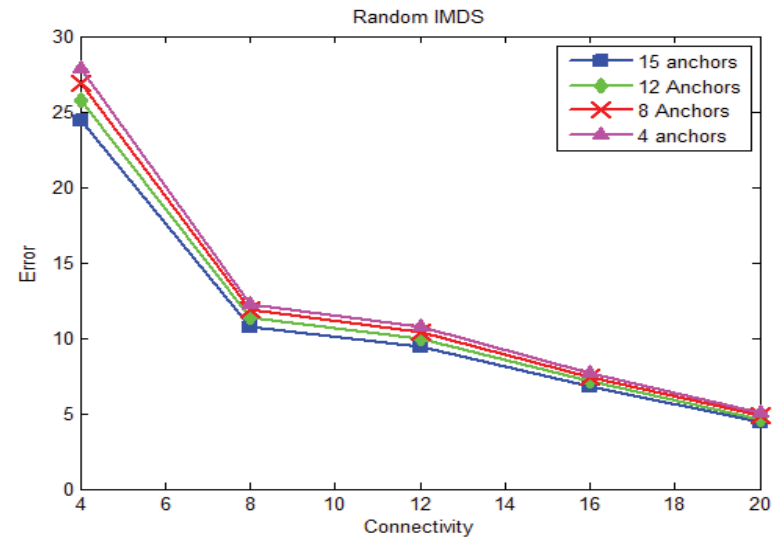

Fig. 8: Impact of number of anchor nodes on estimated error (Random Topology)

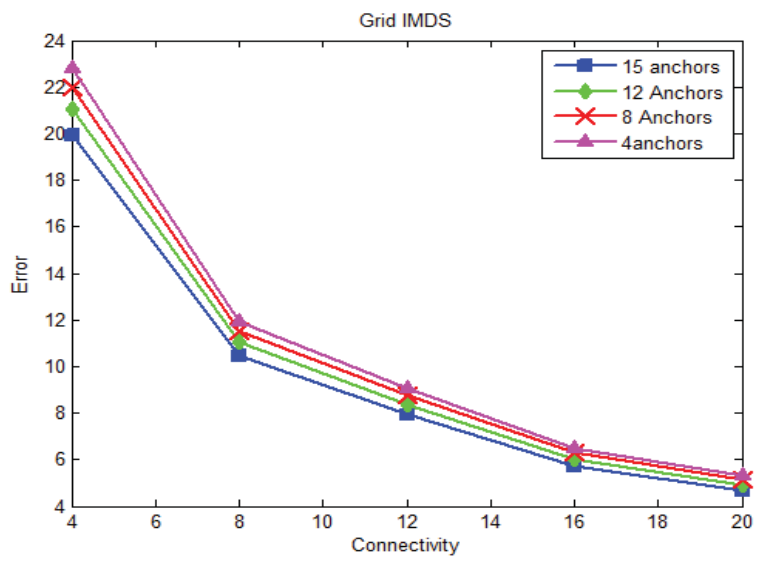

Fig. 8: Impact of number of anchor nodes on estimated error (Grid Topology)

\section{CONCLUSION}

In this paper, we compared MDS- MAP technique of localization with the new algorithm called Improved Multidimensional Scaling (IMDS) in two dimension. We show that IMDS perform better than MDS-MAP and produce acceptable errors due to distance matrix correction.

In future, we plan to work for three dimensional networks and irregular topologies. Also, we plan to analyze the results by introducing clustering in the algorithm.

\section{REFERENCES}

[1] Vishal Garg, Mukul Jhamb, “A Review of Wireless Sensor Network on Localization Techniques,” IJETT, vol. 4 issue 4, pp. 1049-1053, April 2013

[2] S. Tian, X. Zhang, P. Liu, P. Sun, X. Wang, "A RSSI-based DV-hop Algorithm for Wireless Sensor Networks,” IEEE Conference, 2007, pp. $2555-2558$

[3] X. Yingxi, G. Xiang,S. Zeyu and L. Chuanfeng, "WSN Node Localization Algorithm Design Based on RSSI Technology," IEEE Conference on Intelligent Computation And Automation, pp. 556-559, 2012

[4] Y. Shang, W. Ruml, Y.Zhang and M. Fromherz, "Localization from Mere Connectivity," In: Proceedings of ACM MobiHoc. pp. 201-212, 2003

[5] Y. Shang, and W. Ruml, "Improved MDS-based localization," in Twenty-third AnnualJoint Conference of the IEEE Computer and Communications Societies (INFOCOM), pp. 2640-2651, 2004

[6] Tulabandula , Sridhar, "Localization of Wireless Sensor Networks using Multidimensional Scaling," Master's Thesis. Faculty of the Graduate School, University of Missouri-Columbia, 2007

[7] B. R. Stojkoska, and V. Kirandziska, " Improved MDS- based Algorithm for Nodes Localization in Wireless Sensor Networks," IEEE EuroCon, Croatia, 1-4 July 2013, pp. 608-612

[8] S. Patil, and M. Zaveri, "MDS and Trilateration based localization in wireless sensor networks,", doi:10.4236/wsn.2011.36023, SciRp journal, June 2011

[9] G. Yu, and S. Wang, "Hierarchical MDS-based localization Algorithm for wireless sensor networks," $22^{\text {nd }}$ International Conference on Advanced Information Networking and Applications, pp. 748-754, March 2008

[10] V. Vivekanandan, and V. W. Wong, "Ordinal mds-bsed localization for wireless sensor networks," IEEE $64^{\text {th }}$ Vehicular Technology Conference, pp. 1-5, 2006 